\title{
MÉTODO DE BEGGS E BRILL PARA FLUXO MULTIFÁSICO
}

\author{
Jéssica Soares Mota Lôbo ${ }^{1}$, George Simonelli ${ }^{2}$ \\ ${ }^{1}$ Graduanda em Engenharia de Minas - Habilitação Petróleo. Escola Politécnica da \\ UFBA (jessicasmlobo@hotmail.com). Salvador - Brasil. \\ ${ }^{2}$ Professor Assistente da Escola Politécnica da UFBA. \\ Recebido em: 03/10/2016 - Aprovado em: 21/11/2016 - Publicado em: 05/12/2016 \\ DOI: 10.18677/EnciBio_2016B_152
}

\begin{abstract}
RESUMO
Escoamentos multifásicos são encontrados na produção da maioria dos poços de petróleo do mundo. Na maioria dos casos, porém, o escoamento é considerado bifásico, porque considera que o fluxo é formado de líquido e de gás. Nos projetos de produção e transporte é essencial o conhecimento e o domínio do comportamento das vazões, dos padrões de escoamento e do comportamento dinâmico da pressão durante o escoamento multifásico na indústria de petróleo (BEGGS \& BRILL, 1982). Existem correlações empíricas que preveem o comportamento de escoamentos para fluxos monofásicos e multifásicos. Há, porém, na literatura brasileira pouco enfoque na aplicação do método de Beggs e Brill e no seu tratamento de dados. Este trabalho tem por finalidade apresentar uma revisão bibliográfica através de artigos, livros e dissertações de mestrados do modelo de Beggs e Brill para a previsão do comportamento de fluxo multifásicos independente do ângulo de inclinação e o cálculo do gradiente de pressão na coluna de produção. Apesar de ser complexa e envolver o cálculo de muitos parâmetros, a correlação de Beggs e Brill é indispensável na indústria do petróleo. Sua importância é devida a eficiência na previsão do comportamento do escoamento multifásico, levando em consideração as inclinações das tubulações e os padrões de escoamento. Sendo, portanto, muito aplicada no dimensionamento e no comportamento de poços direcionais, nos projetos de elevação artificial, e principalmente, nos projetos de produção.
\end{abstract}

PALAVRAS-CHAVE: Beggs e Brill, Fluxo multifásico, regime de escoamento.

\section{METHOD BEGGS \& BRILL FOR FLOW MULTIPHASE}

\begin{abstract}
Multiphase flows are encountered in almost all producing oil wells. In most cases, however, the flow is considered two-phase liquid-gas because the flow consists of liquid and gas. In the production and transportation projects, the knowledge and mastery over the behavior flow, the flow patterns and the dynamic pressure behavior during the multiphase flow in the oil industry are essential (BEGGS and BRILL, 1982). There are empirical correlations that predict the behavior of flow regime for the Single- Phase and multiphase flows. In Brazil, however, there are a little focus on
\end{abstract}


the application of Beggs and Brill method and its data processing. The present work aims to present a literature review through articles, books and masters dissertations of Beggs and Brill method for predicting the multiphase flow behavior of any tilt angle and the calculation of the pressure gradient in the pipe. Despite being complex and to involve the calculation of many parameters, the correlation of and Beggs e Brill is indispensable in the petroleum industry. Its importance is due to efficient prediction of multiphase flow behavior, taking into account the inclinations of the pipes and flow patterns. Therefore, Beggs and Brill method is very used in the design and behavior of directional wells, in the projects of artificial lift, and in the production projects mainly.

KEYWORDS: Multiphase flow, Beggs and Brill, flow regime.

\section{INTRODUÇÃO}

Escoamentos multifásicos são encontrados em diversos fenômenos, no cotidiano e em vários setores da indústria, por exemplo, na indústria farmacêutica, na indústria de cosméticos, na indústria química e, no foco deste trabalho, na indústria de petróleo. O conhecimento e domínio do comportamento das vazões, dos padrões de escoamento e do comportamento dinâmico da pressão durante o escoamento multifásico são essenciais para os projetos de produção e transporte na indústria de petróleo e em outras indústrias (BEGGS \& BRILL, 1982).

O estudo das propriedades e do comportamento de escoamento é essencial no dimensionamento e previsão do comportamento da produção de petróleo. As fases presentes podem, portanto, estar dispostas em diversas configurações espaciais, que são denominadas de padrões de escoamento (PACHECO et al., 2007). A caracterização dos padrões de escoamento é importante para estimativa das vazões volumétricas, necessária para diversos modelos de escoamento e para o cálculo da variação da pressão nas linhas de produção e de transporte.

Este trabalho tem por finalidade apresentar uma revisão bibliográfica do modelo de Beggs e Brill para a previsão do comportamento do escoamento de fluidos multifásicos com inclinação. Os fluidos utilizados em seus experimentos foram água e ar, variando-se as vazões para diferentes condições de operação (BEGGS \& BRILL, 1982).

\section{REVISÃO LITERÁRIA}

Origem do método de Beggs e Brill

Do início da exploração comercial do petróleo, em 1859 nos Estados Unidos da América, até os dias atuais, as técnicas e os modelos aplicados se aperfeiçoaram muito. Até 1945, os EUA eram o maior produtor do mundo de petróleo. Nos anos 50 aconteceu a intensificação das atividades exploratórias, inclusive exploração offshore. A década de 60 foi marcada pela abundância do petróleo, pelo excesso de produção e pelo consumo desenfreado. O Oriente Médio e a extinta União Soviética quebraram a hegemonia dos EUA com altas reservas de óleo e gás, respectivamente (THOMAS, 2004).

Nos anos 70 aconteceram acentuadas elevações no preço do petróleo, possibilitando novos horizontes de exploração e pesquisas. Entretanto, os EUA perceberam que suas reservas estavam esgotadas e partiram para o aprimoramento 
de métodos de pesquisa. Acontecem, por conseguinte, os grandes avanços e inovações tecnológicas em diversos setores petrolíferos (THOMAS, 2004).

A melhor compreensão da dinâmica do petróleo, a exploração, produção e transporte otimizaram os custos e a produção. Foi nesse cenário que Beggs e Brill publicaram a correlação "Study of Two-Phase Flow in Inclined Pipes" em 1973. Como consequências das pesquisas, as décadas de 80 e 90 são marcadas pela redução nos custos de exploração e produção (THOMAS, 2004).

\section{Escoamento multifásico}

Segundo THOMAS (2004), para que o fluido chegue até os separadores na superfície é necessário que a pressão de fluxo no fundo do poço seja suficiente para vencer a coluna hidrostática do fluído na coluna de produção, as perdas por fricção, as perdas na linha de produção e a pressão dos equipamentos de separação. A produção do óleo pode estar associada com a produção de água, gás livre, gás em solução e, às vezes, areia, configurando, portanto, o fluxo multifásico na coluna de produção. Em busca da otimização dos resultados, verificou-se que os modelos de fluxo monofásico não satisfazem o comportamento do fluxo multifásico. Existem correlações empíricas que preveem o comportamento de escoamentos para fluxos multifásicos.

As correlações empíricas podem: não considerar o deslizamento entre as fases e não considerar o padrão de escoamento no cálculo do fator de atrito (POETTMAN \& CARPENTER, 1952); considerar o deslizamento entre as fases e não considerar o padrão de escoamento no cálculo do fator de atrito e do holdup (HAGEDORN \& BROWN, 1965); considerar o deslizamento entre as fases e considerar o padrão de escoamento no cálculo do fator de atrito e do holdup, ou seja, uma vez estabelecido o padrão de escoamento, as correlações apropriadas para o holdup e para o fator de atrito são determinadas (BEGGS \& BRILL, 1973; GUEDES, 2015).

Os estudos das propriedades e do comportamento de escoamento são essenciais no dimensionamento e na previsão do comportamento da produção de petróleo. As fases água, óleo e gás podem apresentar diversas configurações espaciais, que são denominadas de padrões de escoamento. Porém, Beggs e Brill utilizaram em seus experimentos água e ar, variando-se as vazões para diferentes condições de operação. Portanto, o fluido é bifásico, mas as correlações se estendem e são válidas para o escoamento multifásico (BEGGS \& BRILL, 1973; PACHECO et al., 2007).

Os padrões de escoamentos geralmente citados na literatura do petróleo para escoamento vertical bifásico são quatro: padrão de bolha, padrão de golfadas, de transição e padrão anular conforme mostrados na Figura 1 (TAITEL et al., 1980). E suas características são: 

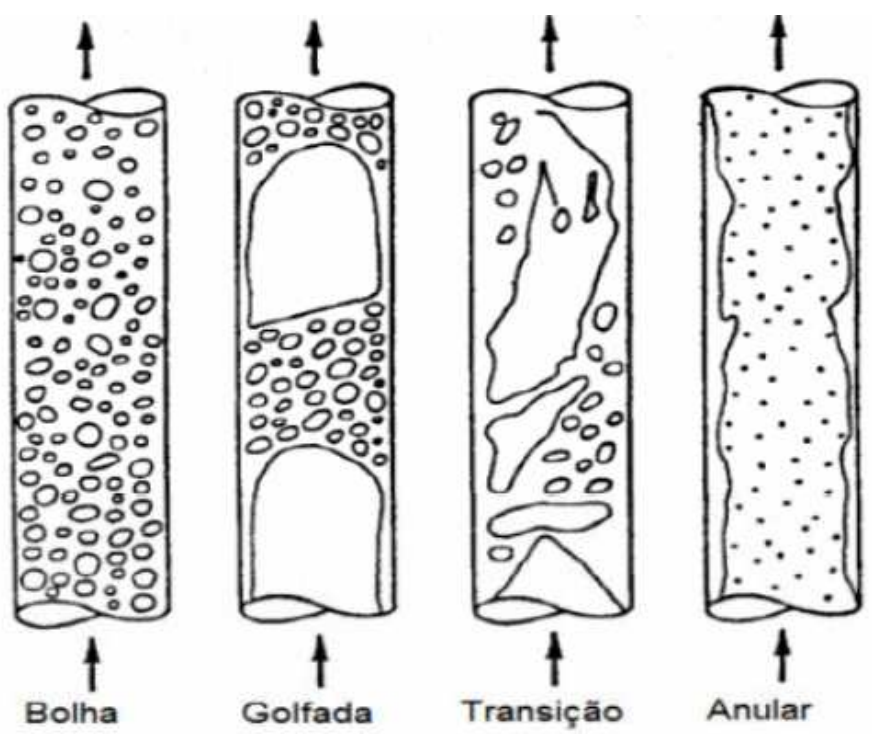

FIGURA 1 - Padrões de escoamento em dutos verticais.

Fonte: BRILL \& MUKHERJEE (1999).

- Padrão de Bolha: A fase gasosa gás está dispersa em forma de pequenas bolhas na fase líquida. As bolhas movem-se com velocidades diferentes, exceto pela densidade, é pouca a influência do gás no gradiente de pressão. A parede do tubo sempre fica em contato com a fase líquida (TAITEL et al., 1980; GUO et al., 2007);

- Padrão de Golfadas: as bolhas de gás se aglutinam em bolhas maiores que, eventualmente, preenchem toda a secção transversal da tubulação. Entre as bolhas grandes de gás estão as golfadas de líquido que contém bolhas menores de gás dissolvido. A velocidade das bolhas de gás é maior que a do líquido. Tanto o gás quanto o líquido têm influência significativa no gradiente de pressão (TAITEL et al., 1980; GUO et al., 2007);

- Padrão de Transição: as grandes bolhas de gás tornam-se instáveis e colapsam, resultando em um padrão de fluxo altamente turbulento com ambas as fases dispersas, não existindo formas características. Ambas as fases têm influência no gradiente de pressão (TAITEL et al., 1980; GUO et al., 2007);

- Padrão Anular: em escoamento anular, o gás torna-se a fase contínua, com o líquido que flui em um anel tubular, o revestimento da superfície do tubo e com gotas arrastadas na fase gasosa. A fase de gás tem influência predominante no gradiente de pressão (TAITEL et al., 1980; GUO et al., 2007).

O holdup do líquido é a relação de volume de uma seção de tubo ocupada por líquido e o volume total dessa seção de tubo. O holdup do líquido pode variar de zero, quando a seção apresenta apenas fluxo de gás, a um, quando a seção apresenta apenas fluxo líquido. O holdup do líquido é medido isolando uma seção entre válvulas de fechamento rápido e medindo o líquido fisicamente capturado. $O$ restante da seção de tubo é ocupado por gás, sendo chamado de holdup do gás. As equações para o holdup do líquido e holdup do gás são, respectivamente, as Equações 1 e 2 (BRILL \& MUKHERJEE,1999; VILLELA, 2004). 


$$
H_{L}=\frac{\text { volume ocupado pelo liquido }}{\text { volume de segmento de tubulação }}
$$

$$
H_{g}=1-H_{L}
$$

$\mathrm{H}_{\mathrm{L}}=$ holdup do líquido, adimensional,

$\mathrm{V}_{\mathrm{L}}=$ volume de uma seção de tubo ocupada por líquido,

$\mathrm{V}=$ volume total dessa seção de tubo,

$\mathrm{H}_{\mathrm{g}}=$ holdup do gás, adimensional.

O holdup do líquido quando não há escorregamento é determinado pela relação do volume de líquido em uma seção de tubo e volume da seção de tubo que existiria, se o gás e o líquido fluíssem à mesma velocidade. O cálculo do holdup do líquido sem escorregamento, representado pela Equação 3, é feito usando as vazões do gás in-situ, $q g$, e do líquido, $q L$.. O holdup do gás sem escorregamento é definido pela Equação 4. (BRILL \& MUKHERJEE,1999; VILLELA, 2004).

$$
\begin{aligned}
& \lambda_{L}=\frac{q_{L}}{q_{L}+q_{g}} \\
& \lambda_{g}=1-\lambda_{L}
\end{aligned}
$$

$\lambda_{\mathrm{L}}=$ holdup do líquido sem escorregamento, adimensional,

$\lambda_{g}=$ holdup do gás, adimensional.

Método de BEGGS e BRILL

BEGGS \& BRILL (1973) desenvolveram correlação capaz de prever o comportamento do escoamento de fluidos multifásicos. A correlação foi desenvolvida através de experimentos com o fluido bifásico formado de água e ar, variando-se as vazões de água até 1.000 barris por dia e de gás 300 mil pés cúbicos por dia. Os testes foram realizados em tubo de acrílico de 1 e 1,5 polegada de diâmetro e comprimento de 90 pés, sustentado por um guincho que fez o tubo variar a inclinação entre -90 e 90 graus, com escoamento descendente e ascendente, respectivamente (BRILL \& MUKHERJEE, 1999).

No modelo de Beggs e Brill, o escoamento vai ser considerado bifásico. Muitas correlações de fluxo bifásico estão baseadas nas variáveis de velocidade superficial e velocidade real. A velocidade superficial de uma fase fluida é definida como a velocidade na qual essa fase estaria sujeita se fluísse só pela seção transversal total do tubo, já a velocidade real é a velocidade que os fluidos apresentam ao escoarem juntos na mesma tubulação.

As próximas equações demonstram os parâmetros de acordo com BEGGS \& BRILL (1973); BEGGS \& BRILL (1982) e BRILL \& MUKHERJEE (1999) para o entendimento do comportamento do fluxo multifásico. A velocidade superficial do gás é calculada pela Equação 5. Já a velocidade real do gás é calculada pela Equação 6: 


$$
\begin{gathered}
v_{s g}=\frac{q_{g}}{A} \\
v_{g}=\frac{q_{g}}{A * H_{g}}
\end{gathered}
$$

A velocidade superficial do líquido é calculada pela Equação 7. Já a velocidade real do líquido é calculada pela Equação 8:

$$
\begin{gathered}
v_{s L}=\frac{q_{L}}{A} \\
v_{L}=\frac{q_{L}}{A * H_{L}}
\end{gathered}
$$

A velocidade superficial da mistura é a soma das velocidades superficiais das fases líquida e gasosa é calculada pela Equação 9:

$$
v_{m}=v_{s g}+v_{s L}
$$

A velocidade de escorregamento é dada pela a diferença entre as velocidades reais das fases gasosa e líquida mostrada na Equação 10:

$$
v_{s}=v_{g}-v_{L}=\frac{v_{s g}}{H_{g}}-\frac{v_{s L}}{H_{L}}
$$

Onde:

$A=a$ área da seção transversal do tubo, $\mathrm{m}^{2}$

$\square s g=$ velocidade superficial do gás, $\mathrm{m} / \mathrm{s}$

$\square \mathrm{g}=$ velocidade real do gás, $\mathrm{m} / \mathrm{s}$

$\square_{\mathrm{sL}}=$ velocidade superficial do líquido, $\mathrm{m} / \mathrm{s}$

$\square \mathrm{L}=$ velocidade real do líquido, $\mathrm{m} / \mathrm{s}$

$\square \mathrm{m}=$ velocidade superficial da mistura, $\mathrm{m} / \mathrm{s}$

$\square_{\mathrm{s}}=$ velocidade de escorregamento, $\mathrm{m} / \mathrm{s}$

Usando as definições anteriores para velocidade, o holdup sem escorregamento, também pode ser calculado como Equação 11:

$$
\lambda_{L}=\frac{v_{s L}}{v_{m}}
$$

A massa específica da mistura multifásica é obtida pela ponderação das massas específicas de cada uma das fases, pode ser calculada pelas Equações 12 e 13:

$$
\begin{gathered}
\rho_{m}=\rho_{L} H_{L(\theta)}+\rho_{G}\left[1-H_{L(\theta)}\right] \\
\rho_{n}=\rho_{L} \lambda_{L}+\rho_{G}\left[1-\lambda_{L}\right]
\end{gathered}
$$

Onde:

$\square \mathrm{L}=$ Densidade do gás, $\mathrm{kg} / \mathrm{m} 3$ 
$\square_{\mathrm{g}}=$ Densidade do líquido, $\mathrm{kg} / \mathrm{m} 3$

$\square_{\mathrm{n}}=$ Densidade da mistura multifásica ponderada por $\lambda_{\mathrm{L}}, \mathrm{kg} / \mathrm{m} 3$

$\mathrm{H}_{L(\theta)}=$ Holdup para determinada inclinação;

$\mathrm{H}_{\mathrm{L}(0)}=$ Holdup em uma tubulação horizontal.

A equação proposta por BEGGS \& BRILL (1973) para estimar a queda de pressão em uma seção de tubulação em estudo é definida pelas Equações 14 e 15:

$$
\begin{gathered}
\frac{d P}{d L}=\frac{\left(\frac{d P}{d L}\right)_{\text {friçã̃o }}+\left(\frac{d P}{d L}\right)_{\text {sleva }}}{1-E_{k}} \\
\frac{d P}{d L}=\frac{f \frac{\rho_{n} v_{m}^{2}}{2 d}+\rho_{m} g \sin \theta}{1-E_{k}}
\end{gathered}
$$

O primeiro componente do numerador está relacionado com a influência do atrito entre as próprias partículas e entre as partículas com a parede da tubulação. Já o segundo termo do numerador refere-se à contribuição devido a elevação do fluido. $O$ termo Ek é chamado de energia cinética adimensional e refere-se a contribuição da queda de pressão pela aceleração, quando muitas vezes é desprezível nos estudos de escoamento (BRILL \& MUKHERJEE, 1999).

$$
E_{k}=\frac{v_{m} v_{S G} \rho_{m}}{P}
$$

BEGGS \& BRILL (1973) prepararam um mapa empírico baseado apenas nos regimes observados em escoamento horizontal. Estes regimes podem ser visualizados na Figura 2. O regime de escoamento correspondente à orientação horizontal da tubulação é determinado pela localização do par $\left(\lambda_{\mathrm{L}}, \mathrm{N}_{\mathrm{Fr}}\right)$. A Figura 3 representa o mapa de regimes de escoamento da correlação. Este mapa é dividido em quatro regiões, regimes Segregados, Transição, Intermitente e Distribuído pelas fronteiras que são os limites de transição.

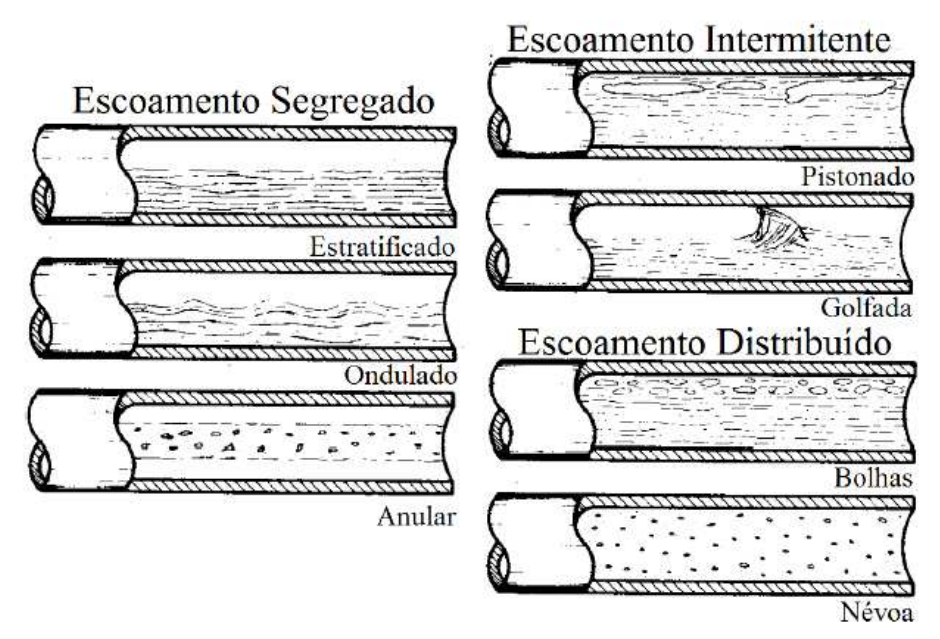


FIGURA 2 - Regimes de escoamento horizontal

Fonte: adaptado de BEGGS \& BRILL (1973).

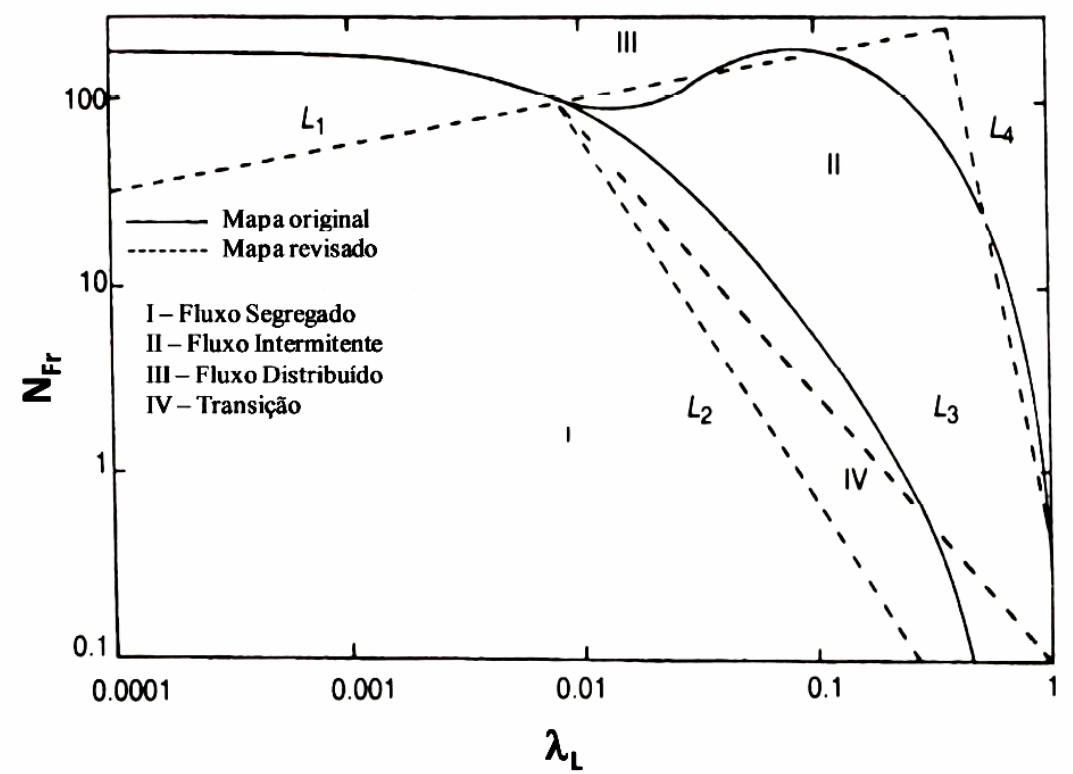

FIGURA 3 - Mapa de padrão escoamento modificado de Beggs e Brill. Fonte: BRILL \& MUKHERJEE (1999).

Os limites de transição são dados pelos números adimensionais L1, L2, L3 e L4 que são calculados pelas Equações 17, 18, 19, 20, respectivamente.

$$
\begin{gathered}
L_{1}=316 \lambda_{L}^{0,302} \\
L_{2}=0,000925 \lambda_{L}^{-2,4684} \\
L_{3}=0,10 \lambda_{L}^{-1,4516} \\
L_{4}=0,5 \lambda_{L}^{-6,738}
\end{gathered}
$$

A identificação do limite de transição pode ser feita através do teste lógico das Equações 21, 22, 23 e 24.

$$
\begin{gathered}
\text { Segregado: } \lambda_{L}<0,01 \wedge N_{F r}<L_{1} \vee \lambda_{L} \geq 0,01 \wedge N_{F r}<L_{2} \\
\text { Transição: } \lambda_{L} \geq 0,01 \wedge L_{2}<N_{F r}<L_{3} \\
\text { Distribuído: } \lambda_{L}<0,4 \wedge N_{F r} \geq L_{1} \vee \lambda_{L} \geq 0,4 \wedge N_{F r}<L_{4} \\
\text { Intermitente: } \lambda_{L} \geq 0,4 \wedge L_{3}<N_{F r}<L_{4} \vee 0,01<\lambda_{L} \geq 0,4 \wedge L_{3}<N_{F r}
\end{gathered}
$$

A equação do holdup líquido para um escoamento horizontal é a mesma para todos os tipos de escoamento, mas os coeficientes empíricos diferenciam de acordo com o tipo de escoamento e é dado pela Equação 25. Os coeficientes empíricos são fornecidos através da Tabela 1 e $\mathrm{N}_{\mathrm{FR}}$ é o número de Froude para mistura. 


$$
\begin{gathered}
H_{L(0)}=\frac{a \lambda_{L}^{b}}{N_{F r}} \\
N_{F r}=\frac{v_{m}^{2}}{g d}
\end{gathered}
$$

TABELA 1 - Coeficientes empíricos para o cálculo do holdup líquido

\begin{tabular}{lccc}
\hline Regime & A & B & C \\
\hline Segregado & 0,980 & 0,4846 & 0,0868 \\
Intermitente & 0,845 & 0,5351 & 0,0173 \\
Distribuído & 1,065 & 0,5824 & 0,0609 \\
\hline
\end{tabular}

Fonte: BEGGS \& BRILL, 1973.

O Holdup líquido inclinado é dado pela Equação 27:

$$
H_{L(\theta)}=\psi * H_{L(0)}
$$

Onde:

$\Psi$ = fator de correlação da inclinação;

E o fator de correlação da inclinação é dado por Equação 28:

$$
\psi=1+C\left[\sin (1,8 \theta)-0,333 \sin ^{3}(1,8 \theta)\right]
$$

Onde,

$\theta=$ Ângulo atual na tubulação;

$\mathrm{C}=$ Parâmetro do holdup líquido.

E o parâmetro do holdup líquido, $C$, é definido pela Equação 29:

$$
C=\left(1-\lambda_{L}\right) \ln \left(e \lambda_{L}^{f} N_{L V}^{g} N_{F r}^{h}\right)
$$

Onde $\mathrm{N}_{\mathrm{LV}}$ é um número adimensional chamado de número de velocidade de líquido e pode ser calculado pela Equação 30:

$$
N_{L V}=1,938 v_{s L} \sqrt[4]{\frac{\rho_{L}}{\sigma_{L}}}
$$

\begin{tabular}{|c|c|c|c|c|}
\hline Configuração do escoamento & $E$ & $\mathbf{F}$ & g & $\mathbf{h}$ \\
\hline Segregado ascendente & 0,011 & $-3,7608$ & 3,5390 & $-1,6140$ \\
\hline Intermitente ascendente & 2,960 & 0,3050 & $-0,4473$ & 0,0978 \\
\hline Distribuído ascendente & \multicolumn{4}{|c|}{$\mathrm{C}=0$ e $\Psi=1$} \\
\hline Descendente & 4,700 & $-0,3692$ & 0,1244 & $-0,5056$ \\
\hline
\end{tabular}

A Tabela 2 representa os coeficientes empíricos para $C \geq 0$ que são determinados como e, f, g e h para o cálculo dos parâmetros do holdup líquido.

TABELA 2 - Coeficientes empíricos para o cálculo dos parâmetros do holdup líquido

Fonte: BEGGS \& BRILL, (1973). 
Quando o padrão de escoamento estiver na região de transição, o holdup líquido deverá ser interpolado entre os padrões de escoamento segregado e de escoamento intermitente e a equação será:

$$
H_{L(\theta)_{\text {transipio }}}=A * H_{L(\theta)_{\text {sggregado }}}+(1-A) * H_{L(\theta)_{\text {intermitents }}}
$$

Sendo:

$$
A=\frac{L_{3}-N_{F r}}{L_{3}-L_{2}}
$$

Correlação para o Fator de Atrito. BEGGS \& BRILL (1982) propuseram a equação para cálculo do fator de fricção para duas fases, que pode ser calculada pelas equações representadas a seguir:

$$
f_{T P}=f_{n}\left(\frac{f}{f_{n}}\right)
$$

Onde $\square$ TP é o fator de fricção bifásico e $\square_{n}$ é o fator de fricção normalizado. 0 fator de fricção normalizado foi determinado utilizando a correlação de CHURCHILL (1977).

$$
\begin{gathered}
f=8\left[\left(\frac{8}{R e}\right)^{12}+\frac{1}{(A+B)^{\frac{3}{2}}}\right]^{\frac{1}{12}} \\
A=\left\{2,457 \ln \left[\frac{1}{\left(\frac{7}{R e}\right)^{0,9}+0,27\left(\frac{\varepsilon}{d}\right)}\right]\right\}^{16} \\
B=\left(\frac{37530}{R e}\right)^{16}
\end{gathered}
$$

Sendo $\square$ =rugosidade relativa.

O número de Reynolds é definido em termos das propriedades físicas da mistura e a equação é dada pela Equação 37 :

$$
N_{R e}=\frac{\rho_{n} v_{m} d}{\mu_{n}}
$$

A relação do fator de fricção bifásico e o fator de fricção normalizado foi obtida experimentalmente e resultou na Equação 38:

$$
\frac{f}{f_{n}}=e^{s}
$$

onde:

$$
\begin{aligned}
& s=\frac{\ln y}{\left[-0,0523+3,182 \ln y-0,8725(\ln y)^{2}+0,01853(\ln y)^{4}\right]} \\
& \mathrm{e}
\end{aligned}
$$




$$
y=\frac{\lambda_{L}}{\left(H_{L(\theta)}\right)^{2}}
$$

Quando $1<\mathrm{y}<1,2$ :

$s=\ln (2,2 y-1,2)$

No método de Beggs e Brill, uma das etapas mais importantes no cálculo do gradiente de pressão é a determinação do regime de escoamento correspondente a orientação horizontal da tubulação e o cálculo do holdup de líquido (TEIXEIRA et al., 2012).

\section{Aplicações}

As correlações empíricas que preveem o comportamento de escoamentos para fluxos monofásicos e multifásicos são usadas na produção dos poços de petróleo do mundo. Devido a complexibilidade no tratamento de dados para fluxo multifásico, a correlação de Beggs e Brill é indispensável na indústria do petróleo. Sendo, portanto, muito aplicada no dimensionamento e no comportamento de poços direcionais, nos projetos de elevação artificial, e principalmente, nos projetos de produção.

Existem, no mercado, diversos simuladores de escoamento multifásico e modelagem para poços de petróleo. Os mais utilizados pelas companhias são: PIPESIM®, PIPEPHASE $\circledast$, OLGA®, LEDAFLOW®, WELLFLO $®$, Traverse. Existe também simulador desenvolvido pela Petrobrás chamado de MARLIM (Multiphase Flow and Artificial Lift Modelling). Este tema é importante para a indústria de petróleo e existe necessidade contínua do aperfeiçoamento das correlações empíricas visando aproximação máxima da realidade (MATOS et al., 2011; SARAH et al., 2014; RAMMAY \& AL-NUAIM, 2015).

A simulação computacional tem sido utilizada em pesquisas em diversas áreas na indústria de petróleo. O uso de processamento de imagens digitais, de modelagem de 2D ou 3D distribuições de fluxo multifásico em poços, estimando holdup líquido para determinados padrões de fluxo em fluxos bifásicos, de modelos matemáticos e de inteligência artificial, como exemplo: Redes Neurais Artificiais, para interpretar os padrões de fluxo e holdup líquido já são feitas como podem ser citados nos trabalhos de JAHANANDISH et al., (2011); AL-SHAMMARI (2011); HASAN \& KABIR (2012); MURAT \& ERTAN (2012); OZBAYOGLU \& YUKSEL, (2012); MIKAMI \& MUKUNOKI (2014); AL-NASER et al., (2015); JEREZCARRIZALES et al., 2015); SILVA \& MARINHO (2016)

\section{CONSIDERAÇÕES FINAIS}

O conhecimento do comportamento de escoamentos multifásicos é fundamental na indústria de petróleo. Este trabalho apresentou uma revisão bibliográfica da correlação de Beggs e Brill para a previsão do comportamento do escoamento de fluxos multifásicos com algumas correções, como: BEGGS \& BRILL (1982); BRILL \& MURHERJEE (1999) e TAITEL et al., (1980). 
Beggs e Brill desenvolveram uma correlação capaz de determinar o comportamento do escoamento multifásico considerando as inclinações das tubulações e os padrões de escoamento, diferenciando-se, portanto, das outras correlações empíricas, tornando-a essencial na indústria de petróleo. Existem muitos estudos sobre o comportamento de fluxo multifásico, conferindo, assim, uma diversa bibliografia sobre as correlações de fluxo multifásico e suas aplicações. Contudo, na literatura brasileira é possível o maior aprofundamento nesse tema, apesar das teses de mestrado e doutorado abordados neste artigo.

Apesar das limitações nos procedimentos experimentais, como, por exemplo, o fluxo ser bifásico e ter as vazões limitadas, muitos trabalhos já existentes validam o uso do método de Beggs e Brill para ser usado amplamente para fluxo multifásico e para altas vazões. Estudos para a otimização da produção já foram feitos inclusive em campos maduros e o comportamento com o óleo com alta viscosidade como podem ser citados os trabalhos de NASCIMENTO et al., (2011); ZHAO et al., (2013).

Apesar de ser complexa e haver o cálculo de muitos parâmetros, a correlação de Beggs e Brill, em comparação às outras correlações já existentes, é considerada a mais precisa. Sendo muito aplicada, portanto, nos projeto elevação artificial, aplicada no dimensionamento e no comportamento de poços direcionais, e principalmente, nos projetos de produção.

\section{REFERÊNCIAS}

AL-NASER, M.; ELSHAFEI, M.; AL-SARKHI, A. Two-Phase Flow Regimes Identification using Artificial Neural Network with Nonlinear Normalization. Proceedings of the 2nd International Conference on Fluid Flow, Heat and Mass Transfer. Paper No. 133. Canada. 2015

AL-SHAMMARI, A. Accurate prediction of pressure drop in two-phase vertical flow systems using artificial intelligence. SPE/DGS Saudi Arabia Section Technical Symposium and Exhibition held in Al-Khobar Saudi Arabia; paper SPE 149035 2011. http://dx.doi.org/10.2118/149035-MS

BEGGS, H.D.; BRILL, J.P. Two-Phase Flow in Pipes, 4th ed., University of Tulsa. Printer, USA, 1982.

BEGGS, H. D. AND BRILL, J. P., Study of Two-phase Flow in Inclined Pipes, Journal of Petroleum Technology, p. 607-617, 1973.

BRILL, J.P., MUKHERJEE, H. Multiphase Flow in Wells, Monograph Series, SPE, Richardson, Texas 17. 1999.

CHURCHILL, S., Friction-Factor Equation Spans all Fluid-Flow Regimes, Chemical Engineering, 1977.

GUEDES, THAMIRES A. L. Modelagem Do Escoamento Em Linhas De Produção Offshore Com Garantia De Escoamento. Dissertação de Mestrado. Programa de Pós-graduação em Engenharia Química. Universidade Federal do Rio de Janeiro, 2015. 
GUO, B; LYONS, W. C.; GHALAMBOR A. Petroleum Production Engineering: a computer-assisted approach. Oxford: Elsevier, 2007.

HAGEDORN, A. R.; BROWN, K. E. Experimental Study of Pressure Gradients Occuring During Continuous Two-Phase Flow in Small-Diameter Vertical Conduits. Journal of Petroleum Technology, p. 475-484, Abr/1965.

HASAN, A. R.; KABIR,C. S. Wellbore heat-transfer modeling and applications. Journal of Petroleum Science and Engineering, v. 86-87, p 127-136, 2012. http://dx.doi.org/10.1016/j.petrol.2012.03.021223

JAHANANDISH, I.; SALIMIFARD,B.; JALALIFAR, H. Predicting bottomhole pressure in vertical multiphase flowing wells using artificial neural networks. Journal of Petroleum Science and Engineering, v. 75, Issues 3-4, p 336-342, 2011.

http://dx.doi.org/10.1016/j.petrol.2010.11.019

JEREZ-CARRIZALES, M., JARAMILLO, J. E. AND FUENTES, D. Prediction of Multiphase Flow in Pipelines: Literature Review. Ingeniería y Ciencia, v. 11, n. 22, p. 213-233, 2015. http://www.scielo.org.co/pdf/ince/v11n22/v11n22a10.pdf

MATOS, A., ALVES, A. C., NASCIMENTO, C. M. B. M., PRINCE, J. A. P. Comparison of Different Flow Simulators for Designing Deep-water Oil Pipelines Convergent Network. Rio Pipeline Conference \& Exposition. Rio de Janeiro, 2011.

MIKAMI, K.; MUKUNOKI, T. Study On Mechanism Of Two-phase Flow In Porous Media Using Micro Focused X-ray CT. International Petroleum Technology Conference. Qatar. 2014. http://dx.doi.org/10.2523/IPTC-17676-MS

MURAT, A., ERTAN, H. Analysis of Gas-Liquid Behavior in Eccentric Horizontal Annuli with Image Processing and Artificial Intelligence Techniques. Journal of Petroleum Science and Engineering, v. 81, p. 31-40. 2012. http://dx.doi.org/10.1016/j.petrol.2011.12.008

NASCIMENTO, J. C. S.; NOGUEIRA DUARTE, L.J.; SANTOS, L. C. L. Optimization of the Oil and Gas Flow in Mature Fields. In: RIO PIPELINE CONFERENCE AND EXPOSITION, 2011, Rio de Janeiro - RJ. Rio Pipeline, v. 5, 2011.

PACHECO, F.; CAVALCANTE, C.C.P.; BANNWART, A.C.; MENDES, J. R. P.; SERAPIÃO, ADRIANE B. S. Identificação de Padrões de Escoamento Multifásico e Determinação da Perda de Carga com o Auxílio de Redes Neurais. In: 40. Congresso Brasileiro de P\&D em Petróleo e Gás (PDPetro), 2007, Campinas. Anais do 40. Congresso Brasileiro de P\&D em Petróleo e Gás, 2007.

POETTMAN, F. H.; CARPENTER, P. G. The Multiphase Flow of Gas, Oil, and Water Through Vertical Flow Strings with Application to the Design of Gas-lift Installatios. American Petroleum Institute, Nova York, v. 257, Janeiro 1952. 
OZBAYOGLU, A. M; YUKSEL, H. E. Analysis of gas-liquid behavior in eccentric horizontal annuli with image processing and artificial intelligence techniques. Journal of petroleum science and engineering, 81, 31-40. 2012.

http://dx.doi.org/10.1016/j.petrol.2011.12.008

RAMMAY, M. H.; AL-NUAIM, S. Flow Regime Prediction Using Fuzzy Logic And Modification In Beggs And Brill Multiphase Correlation. International Petroleum Technology Conference. Qatar. 2015. http://dx.doi.org/10.2523/IPTC-18267-MS

SARAH, A. A.; JULIOS, U. A.; MARY-ANN, O. Pressure Gradient Prediction of Multiphase Flow in Pipes. British Journal of Applied Science \& Technology, v. 4, p. 4945-4958, 2014.http://dx.doi.org/10.9734/BJAST/2014/12985

SILVA, L. D. S.; MARINHO, J. L. G. Study on pressure drop and liquid volume fraction of the oil-gas flow in a vertical pipe using cfx and the beggs and brill correlation: viscosity effects. Brazilian Journal Of Petroleum And Gas. v. 10, n. 1, p. 001-008, 2016. http://dx.doi.org/10.5419/bjpg2016-0001

TAITEL, Y. M., BARNEA, D., DURKER A.E. Modeling Flow Pattern Transitions for Upward Gas-Liquid Flow in Vertical Tubes. AIChE, 1980.

TEIXEIRA, R. G. D.; SECCHI, A. R.; BISCAIA JUNIOR, Evaristo Chalbaud . Otimização Econômica do Diâmetro Interno de Tubulações em Problemas de Escoamento Bifásico Líquido / Vapor. In: XIX Congresso Brasileiro de Engenharia Química, 2012, Búzios. Anais do Congresso Brasileiro de Engenharia Química, v. 1. p. 69-78, 2012.

THOMAS, J. E. (Org.) Fundamentos de Engenharia de Petróleo. 2 ed. Rio de Janeiro: Interciência, 2004.

VILLELA, M. J. R. Análise do comportamento da temperatura em sistemas de produção de petróleo: comparação entre completação seca e molhada. Dissertação de mestrado. Pontifícia Universidade Católica do Rio De Janeiro, 2004.

ZHAO, Y.; YEUNG, H.; ZORGANI, E.; ARCHIBONG, A.; LAO, L. High viscosity effects on characteristics of oil and gas two-phase flow in horizontal pipes. Chemical Engineering Science, v. 95, p. 343-352, 2013.

Available: http://dx.doi.org/10.1016/i.ces.2013.03.004 225 